\title{
Lattice Boltzmann simulations in microfluidics: probing the no-slip boundary condition in hydrophobic, rough, and surface nanobubble laden microchannels
}

\author{
Jens Harting $\cdot$ Christian Kunert · Jari Hyväluoma
}

Received: 14 August 2009/Accepted: 7 September 2009/Published online: 14 October 2009

(C) The Author(s) 2009. This article is published with open access at Springerlink.com

\begin{abstract}
In this contribution, we review recent efforts on investigations of the effect of (apparent) boundary slip by utilizing lattice Boltzmann simulations. We demonstrate the applicability of the method to treat fundamental questions in microfluidics by investigating fluid flow in hydrophobic and rough microchannels as well as over surfaces covered by nano- or microscale gas bubbles.
\end{abstract}

Keywords Apparent and intrinsic slip .

Rough and hydrophobic surfaces .

Lattice Boltzmann simulations

\section{Introduction}

During the past few decades, the miniaturization of technical devices down to submicrometric sizes has made considerable progress. In particular, the so-called microelectro-mechianical systems (MEMS) became available for chemical, biological, and technical applications leading to

J. Harting $(\square)$

Department of Applied Physics, TU Eindhoven, Den Dolech 2, 5600MB Eindhoven, The Netherlands

e-mail: j.harting@tue.nl

J. Harting · C. Kunert

Institute for Computational Physics, University of Stuttgart, Pfaffenwaldring 27, 70569 Stuttgart, Germany

e-mail: kuni@icp.uni-stuttgart.de

C. Kunert

e-mail: kuni@icp.uni-stuttgart.de

J. Hyväluoma

Department of Physics, University of Jyväskylä,

40014 Jyväskylä, Finland

e-mail: jari.hyvaluoma@jyu.fi the rise of "microfluidics" about 20 years ago (Tabeling 2005). A wide variety of microfluidic systems including gas chromatography systems, electrophoretic separation systems, micromixers, DNA amplifiers, and chemical reactors were developed. Next to those "practical applications," microfluidics was used to answer fundamental questions in physics including the behavior of single molecules or particles in fluid flow or the validity of the no-slip boundary condition (Tabeling 2005; Lauga et al. 2005). The latter is the focus of the current review and is investigated in detail by mesoscopic computer simulations.

Reynolds numbers in microfluidic systems are usually small, i.e., usually below 0.1 . In addition, due to the small scales of the channels, the surface-to-volume ratio is high causing surface effects such as wettability or surface charges to be more important than in macroscopic systems. Also, the mean free path of a fluid molecule might be of the same order as the characteristic length scale of the system. For gas flows, this effect can be characterized by the so-called Knudsen number (Knudsen 1909). While the Knudsen number provides a good estimate for when to expect rarefaction effects in gas flows, for liquids one would naively assume that its velocity close to a surface always corresponds to the actual velocity of the surface itself. This assumption is called the no-slip boundary condition and can be counted as one of the generally accepted fundamental concepts of fluid mechanics. However, this concept was not always well accepted. Some centuries ago, there were long debates about the velocity of a Newtonian liquid close to a surface, and the acceptance of the no-slip boundary condition was mostly due to the fact that no experimental violations could be found, i.e., the so-called boundary slip could not be detected.

In recent years, it became possible to perform very well controlled experiments that have shown a violation of the no-slip boundary condition in submicron-sized geometries. 
Since then, mostly, not only experimental (Lauga et al. 2005; Craig et al. 2001; Tretheway and Meinhart 2004; Cheng and Giordano 2002; Choi et al. 2003; Baudry and Charlaix 2001; Cottin-Bizonne et al. 2002; Vinogradova and Yakubov 2003), but also theoretical studies (Vinogradova 1995; Gennes 2002) as well as computer simulations (Succi 2002; Barrat and Bocquet 1999; Cieplak et al. 2001; Thompson and Troian 1997; Tretheway et al. 2002) have been performed to improve our understanding of boundary slip. The topic is of fundamental interest because it has practical consequences in the physical and engineering sciences as well as for medical and industrial applications. Interestingly, also for gas flows, often a slip length much larger than expected from classical theory can be observed. Extensive reviews of the slip phenomenon have recently been published by Lauga et al. (2005), Neto et al. (2005), as well as Bocquet and Barrat (2007).

The reason for our unsatisfactory understanding of boundary slip is that the behavior of a fluid close to a solid interface is very complex and involves the interplay of many physical and chemical properties. These include the wettability of the solid, the shear rate or flow velocity, the bulk pressure, the surface charge, the surface roughness, as well as impurities and dissolved gas. Because all those quantities have to be determined very precisely, it is not surprising that our understanding of the phenomenon is still very unsatisfactory. Owing to the large number of different parameters, a significant dispersion of the results can be observed for almost similar systems (Lauga et al. 2005; Neto et al. 2005). For example, observed slip lengths vary between a few nanometers (Churaev et al. 1984) and micrometers (Tretheway and Meinhart 2004) and while some authors find a dependence of the slip on the flow velocity (Craig et al. 2001; Choi et al. 2003; Zhu and Granick 2001), others do not (Tretheway and Meinhart 2004; Cheng and Giordano 2002).

A boundary slip is typically quantified by the so-called slip length $\beta-$ a concept that was already proposed by Navier in 1823 . He introduced a boundary condition where the fluid velocity at a surface is proportional to the shear rate at the surface (Navier 1823) (at $x=x_{0}$ ), i.e.,

$v_{z}\left(x_{0}\right)=\beta \frac{\partial v_{z}(x)}{\partial x}$

In other words, the slip length $\beta$ can be defined as the distance from the surface where the relative flow velocity vanishes. Assuming a typical Poiseuille setup consisting of a pressure-driven flow of an incompressible liquid between two infinite planes, the velocity in flow direction $\left(v_{z}\right)$ at position $x$ between the planes is given by

$v_{z}(x)=\frac{1}{2 \mu} \frac{\partial P}{\partial z}\left[d^{2}-x^{2}-2 d \beta\right]$ where $2 d$ is the distance between the planes, and $\mu$ is the dynamic viscosity. $\partial P / \partial z$ is the pressure gradient. In contrast to a no-slip formulation, the last term in Eq. 2 linearly depends on the slip length $\beta$.

Most recent computer simulations apply molecular dynamics and report increasing slip with decreasing liquid density (Koplik et al. 1989; Thompson and Robbins 1990) or liquid-solid interactions (Cieplak et al. 2001; Nagayama and Cheng 2004), while slip decreases with increasing pressure (Barrat and Bocquet 1999). These simulations are usually limited to a few tens of thousand particles, length scales of a few nanometers and time scales of nanoseconds. Also, shear rates are usually some orders of magnitude higher than in any experiment (Lauga et al. 2005). Owing to the small accessible time and length scales of molecular dynamics simulations, mesoscopic simulation methods, such as the lattice Boltzmann method, are well applicable for the simulation of microfluidic experiments.

The experimental investigation of apparent slip can be based on different setups: a fluid is pumped through a microchannel, and the measured mass flow rate at the end of the channel is compared to the theoretical value with no-slip boundary conditions. From the deviation of the two values, the magnitude of slip can be computed (Tretheway and Meinhart 2002). Another possibility is to measure the slip length directly using optical methods such as particle image velocimetry (PIV). Very popular is the modification of an atomic force microscope (AFM) by adding a silicon sphere to the tip of the cantilever. While moving the sphere toward the boundary, the required force is measured. It is possible to measure the amount of slip at the wall by comparing the force needed to move the sphere with its theoretical value (Vinogradova and Yakubov 2003; Vinogradova 1996).

During the past few years, the substantial scientific research invested in the slip phenomenon has led to a more clear picture which can be summarized as follows: one can argue that many surprising results published were only due to artifacts or misinterpretation of experiments. In general, there seems to be an agreement within the community that slip lengths larger than a few nanometers can usually be referred to as "apparent slip" and are often caused by experimental artifacts. Small slip lengths are experimentally even harder to determine and require sophisticated setups such as the modified AFMs as described above. Here, small variations of the apparatus such as choosing a different shape of the cantilever or modifying the control circuit of the sample holder can lead to substantial variation of the measurements. Also, the theoretical equations correlating the measured force to the slip length are only valid for perfect surfaces and infinitely slow oscillations of the sphere. Therefore, it is of importance to perform 
computer simulations which have the advantage that most parameters can be changed independently without modifying anything else. Thus, the influence of every single modification can be studied to present estimates of expected slip lengths.

\section{Apparent slip in hydrophobic microchannels}

The simulation method used to study microfluidic devices has to be chosen carefully. While Navier-Stokes solvers are able to cover most problems in fluid dynamics, they lack the possibility to include the influence of molecular interactions as needed to model boundary slip. Molecular dynamics (MD) simulations are the best choice to simulate the fluidwall interaction, but the computer power today is not sufficient to simulate length and time scales necessary to achieve orders of magnitude which are relevant for experiments. However, boundary slip with a slip length $\beta$ of the order of many molecular diameters $\sigma$ has been studied with molecular dynamics simulations by various authors (Baudry and Charlaix 2001; Cieplak et al. 2001; Thompson and Troian 1997; Cottin-Bizonne et al. 2004; Priezjev et al. 2005).

This article focuses on numerical investigations of the slip phenomenon by means of lattice Boltzmann simulations. While an emphasis is put on reviewing our own contributions to the field, the achievements of other groups are commonly referred to. However, it should be noticed that while a large number of groups utilizes the lattice Boltzmann technique to investigate microfluidic problems, only a very small number of researchers are actually applying the method to studying slippage. Even though interactions have to be described on a mesoscopic scale, this is surprising since mesoscopic simulation methods offer a closer relation to experimentally relevant time and length scales than microscopic techniques such as molecular dynamics.

In the lattice Boltzmann method, one discretizes the Boltzmann kinetic equation

$\left[\frac{\partial}{\partial t}+v \nabla_{x}\right] \eta(\mathbf{x}, \mathbf{v}, t)=\mathbf{\Omega}$

on a lattice. The Boltzmann kinetic equation describes the evolution of the single particle probability density $\eta(\mathbf{x}, \mathbf{v}, t)$, where $\mathbf{x}$ is the position, $\mathbf{v}$ the velocity, and $t$ the time. The derivatives represent simple propagation of a single particle in real and velocity space, whereas the collision operator $\boldsymbol{\Omega}$ takes into account molecular collisions in which a particle changes its momentum due to a collision with another particle. In order to represent the correct physics, the collision operator should conserve mass and momentum, and should be Galilei invariant. By performing a Chapman Enskog procedure, it can be shown that such a collision operator $\boldsymbol{\Omega}$ reproduces the Navier-Stokes equation (Succi 2001). In the lattice Boltzmann method, the time $t$, the position $\mathbf{x}$, and the velocity $\mathbf{v}$ are discretized.

A few groups have applied the lattice Boltzmann method for the simulation of microflows and to study boundary slip. A popular approach is to introduce slip by generalizing the no-slip bounce-back boundary conditions to allow specular reflections with a given probability (Succi 2002; Tretheway et al. 2002; Tang et al. 2005; Sbragaglia and Succi 2005), or to apply diffuse scattering (Ansumali and Karlin 2002; Sofonea and Sekerka 2005; Niu et al. 2004). It has been shown by Guo et al. that these approaches are virtually equivalent (Guo et al. 2007). Another possibility is to modify the fluid's viscosity, i.e., the fluid viscosity is modified due to local density variations to model slip (Nie et al. 2002). In both cases, the parameters determining the properties at the boundaries are "artificial" parameters, and they do not have any obvious physical meaning. Therefore, they are not easily mappable to experimentally available values. We model the interaction between hydrophobic channel walls and the fluid by means of a multiphase lattice Boltzmann model. Our approach overcomes this problem by applying a mesoscopic force between the walls and the fluid. A similar approach is used by Zhu et al. (2005), Benzi et al. (2006a), and Zhang et al. (2004). This force applied at the boundary can be linked to the contact angle which is commonly used by experimentalists to quantitatively describe the wettability of a material (Benzi et al. 2006b; Huang et al. 2007).

The simulation method and our implementation of boundary conditions are described as follows. A multiphase lattice Boltzmann system can be represented by a set of equations

$\eta_{i}^{\alpha}\left(\mathbf{x}+\mathbf{c}_{i}, t+1\right)-\eta_{i}^{\alpha}(\mathbf{x}, t)=\Omega_{i}^{\alpha}, \quad i=0,1, \ldots, b$,

where $\eta_{i}^{\alpha}(\mathbf{x}, t)$ is the single-particle distribution function, indicating the amount of species $\alpha$ with velocity $\mathbf{c}_{i}$, at site $\mathbf{x}$ on a D-dimensional lattice of coordination number $b$ (D3Q19 in our implementation), at time-step $t$. This is a discretized version of Eq. 3 without external forces $\mathbf{F}$ for a number of species $\alpha$. For the collision operator $\Omega_{i}^{\alpha}$ we choose the Bhatnagar-Gross-Krook (BGK) form (Bhatnagar et al. 1954)

$\mathbf{\Omega}_{i}^{\alpha}=-\frac{1}{\tau^{\alpha}}\left(\eta_{i}^{\alpha}(\mathbf{x}, t)-\eta_{i}^{\alpha e q}\left(\mathbf{u}^{\alpha}(\mathbf{x}, t), \eta^{\alpha}(\mathbf{x}, t)\right)\right)$,

where $\tau^{\alpha}$ is the mean collision time for component $\alpha$ and determines the kinematic viscosity

$v^{\alpha}=\frac{2 \tau^{\alpha}-1}{6}$.

of the fluid. The relaxation time $\tau^{\alpha}$ is kept constant at 1.0 in this study. The system relaxes to an equilibrium distribution 
$\eta_{i}^{\alpha}{ }^{e q}$ which can be derived imposing restrictions on the microscopic processes, such as explicit mass and momentum conservation for each species. In our implementation, we choose for the equilibrium distribution function

$\eta_{i}^{e q}=\zeta_{i} \eta^{\alpha}\left[1+\frac{\mathbf{c}_{i} \cdot \mathbf{u}}{c_{s}^{2}}+\frac{\left(\mathbf{c}_{i} \cdot \mathbf{u}\right)^{2}}{2 c_{s}^{4}}-\frac{u^{2}}{2 c_{s}^{2}}+\frac{\left(\mathbf{c}_{i} \cdot \mathbf{u}\right)^{3}}{6 c_{s}^{6}}-\frac{u^{2}\left(\mathbf{c}_{i} \cdot \mathbf{u}\right)}{2 c_{s}^{4}}\right]$,

which is a polynomial expansion of the Maxwell distribution. $\mathbf{c}_{i}$ 's are the velocity vectors pointing to neighboring lattice sites and $\zeta_{i}$ are the lattice weights resulting from the velocity space discretization. $c_{s}=1 / \sqrt{3}$ is the speed of sound for the D3Q19 lattice. The macroscopic values can be derived from the single-particle distribution function $\eta_{i}^{\alpha}(\mathbf{x}, t)$, i.e., the density $\eta^{\alpha}(\mathbf{x}, t)$ of the species $\alpha$ at lattice site $\mathbf{x}$ is the sum over the distribution functions $\eta_{i}^{\alpha}(\mathbf{x}, t)$ for all lattice velocities $\mathbf{c}_{i}$

$\eta^{\alpha}(\mathbf{x}, t) \equiv \sum_{i} \eta_{i}^{\alpha}(\mathbf{x}, t)$.

$\mathbf{u}^{\alpha}(\mathbf{x}, t)$ is the macroscopic velocity of the fluid, defined as

$\eta^{\alpha}(\mathbf{x}, t) \mathbf{u}^{\alpha}(\mathbf{x}, t) \equiv \sum_{i} \eta_{i}^{\alpha}(\mathbf{x}, t) \mathbf{c}_{i}$.

Interactions between different fluid species are introduced, according to Shan and Chen, as a mean field body force between nearest neighbors (Shan and Chen 1993, 1994),

$\mathbf{F}^{\alpha}(\mathbf{x}, t) \equiv-\psi^{\alpha}(\mathbf{x}, t) \sum_{\bar{\alpha}} g_{\alpha \bar{\alpha}} \sum_{\mathbf{x}^{\prime}} \psi^{\bar{\alpha}}\left(\mathbf{x}^{\prime}, t\right)\left(\mathbf{x}^{\prime}-\mathbf{x}\right)$,

where $\psi^{\alpha}(\mathbf{x}, t)=\left(1-e^{-\eta^{\alpha}(\mathbf{x}, t) / \eta_{0}}\right)$ is the so-called effective mass with $\eta_{0}$ being a reference density that is set to 1 in our case (Shan and Chen 1993). $g_{\alpha \bar{\alpha}}$ is a force coupling constant, whose magnitude controls the strength of the interaction between component $\alpha$ and $\bar{\alpha}$. The dynamic effect of the force is realized in the BGK collision operator (5) by adding an increment $\delta \mathbf{u}^{\alpha}=\tau^{\alpha} \mathbf{F}^{\alpha} / \eta^{\alpha}$ to the velocity $\mathbf{u}$ in the equilibrium distribution function (7). A repulsive potential between surface and fluid can be used to model hydrophobic fluidsurface interactions. Such a potential is realized by attaching the imaginary fluid "density" $\eta^{\text {wall }}$ to the first lattice site inside the wall. Only the distribution corresponding to the rest velocity is filled, while the remaining ones are kept at 0 . As a result, the only difference between $\eta^{\text {wall }}$ and any other fluid packages on the lattice $\eta^{\bar{\alpha}}$ is that the fluid corresponding to $\eta^{\text {wall }}$ is taken into account only for the collision step and for the calculation of Eq. 10, but not in the propagation step. Therefore, we can adopt $\eta^{\text {wall }}$ and the coupling constant $g_{\alpha, \text { wall }}$ to tune the fluid-wall interaction. $g_{\alpha \text {,wall }}$ is kept at 0.08 throughout this article if not mentioned otherwise, and all the values are reported in lattice units. These parameters allow to simulate a wide range of effective interactions without compromising on numerical stability. In addition, we apply second-order correct mid-grid bounce-back boundary conditions between the fluid and the surface which assures vanishing velocities at solid surfaces. Here, a distribution function that would be advected into a solid node is simply reversed and advected into the opposite direction (Succi 2001).

From molecular dynamics simulations, it is known that the fluid-wall interactions causing a slip phenomenon usually take place within a few molecular layers of the liquid along the boundary surface (Baudry and Charlaix 2001; Cieplak et al. 2001; Thompson and Troian 1997; Cottin-Bizonne et al. 2004). Our coarse-grained fluid-wall interaction acts on the length scale of one lattice constant and does not take the molecular details into account. Therefore, coarse-grained implementations based on the lattice Boltzmann method are only able to reproduce an averaged effect of the interaction and cannot fully resolve the correct flow profile very close to the wall and below the resolution of a single lattice spacing. However, in order to understand the influence of the hydrophobicity on experimentally observed apparent slip, it is fully sufficient to investigate the flow behavior on more macroscopic scales as they are accessible for experimental investigation. Coarsegrained interaction models could be improved by a direct mapping of data obtained from MD simulations to the coupling constant $g_{\alpha, \text { wall }}$ allowing a direct comparison of the influence of liquid-wall interactions on the detected slip (Harting et al. 2006). Similar approaches are known from quantitative comparisons of lattice Boltzmann and molecular dynamics simulations in the literature (Horbach and Succi 2006; Chibbaro et al. 2008).

The simulations in this study use a setup of two infinite planes separated by the distance $2 d$. We call the direction between the two planes $x$, and if not stated otherwise, $2 d$ is set to 64 lattice sites. In $y$ direction, we apply periodic boundary conditions. Here, eight lattice sites are sufficient to avoid finite size effects since there is no propagation in this direction. $z$ is the direction of the flow with our channels being 512 lattice sites long. At the beginning of the simulation $(t=0)$, the fluid is at rest. We then apply a pressure gradient $\nabla P$ in the $z$-direction to generate a planar Poiseuille flow. Assuming Navier's boundary condition, the slip length $\beta$ is measured by fitting the theoretical velocity profile as given by Eq. 2 in flow direction $\left(v_{z}\right)$ at position $x$, to the simulated data via the slip length $\beta$. We validate this approach by comparing the measured mass flow rate $\int \eta v(x) \mathrm{dx}$ to the theoretical mass flow without boundary slip and find a very good agreement. The dynamic viscosity $\mu$ as well as the pressure gradient $\frac{\partial P}{\partial z}$ needed to fit Eq. 2 are obtained from our simulation data.

In Harting et al. (2006), we show that this model creates a larger slip $\beta$ with stronger interaction, namely, larger $g_{\alpha, \text { wall }}$ and larger $\eta^{\text {wall }}$. The maximum available slip length 
measured is 5.0 in lattice units. For stronger repulsive potentials, the density gradient at the fluid-wall interface becomes too large, causing the simulation to become unstable. At lower interactions, the method is very stable, and the slip length $\beta$ is independent of the distance $d$ between the two plates and, therefore, independent of the resolution. We also show that the slip decreases with increasing pressure since the relative strength of the repulsive potential compared to the bulk pressure is weaker at high pressure. Therefore, the pressure reduction near the wall is less in the high pressure case than in the low pressure one. Furthermore, we demonstrate that $\beta$ can be fitted with a semianalytic model based on a two-viscosity model.

We study the dependence of the slip length $\beta$ on the flow velocity for a wide range of velocities of more than three decades as shown in Fig. 1 and in Harting et al. (2006). In the figure, we show data for different fluid-wall interactions $0<\eta^{\text {wall }}<2.0$ and flow velocities from $10^{-4}<v<10^{-1}$. For simplicity, we restrict ourselves to $g_{\alpha, \text { wall }}=0.08$ which is a suitable value found from parameter studies given in Harting et al. (2006). Within this region, we confirm the findings of many steady-state experiments (Cheng and Giordano 2002), namely, the slip length is independent of the flow velocity and only depends on the wettability of the channel walls. Some dynamic experiments, however, find a shear rate-dependent slip (Zhu and Granick 2001; Neto et al. 2003). These experiments often utilize a modified AFM, as described in the introduction, to detect boundary slippage. Since the slip length is found to be constant in our simulations after sufficiently long simulation times, we cannot confirm these results. However, it has been proposed by various authors that this velocity dependence is due to noncontrolled effects such as impurities or surface nanobubbles. In simulations, we can only find a shear rate dependence if the system has not yet reached the steady state or if time-dependent accelerations are present (Kunert and Harting 2008a).

Our mesoscopic approach is able to reach the small flow velocities of known experiments, and reproduces results from experiments and other computer simulations, namely, an increase of the slip with increasing liquid-solid interactions, the slip being independent of the flow velocity, and a decreasing slip with increasing bulk pressure. In addition, within our model, we develop a semianalytic approximation of the dependence of the slip on the bulk pressure as described in Harting et al. (2006).

\section{Roughness induced apparent slip}

If typical length scales of the experimental system are comparable to the scale of surface roughness, the effect of roughness cannot be neglected anymore. Figure 2 (left)

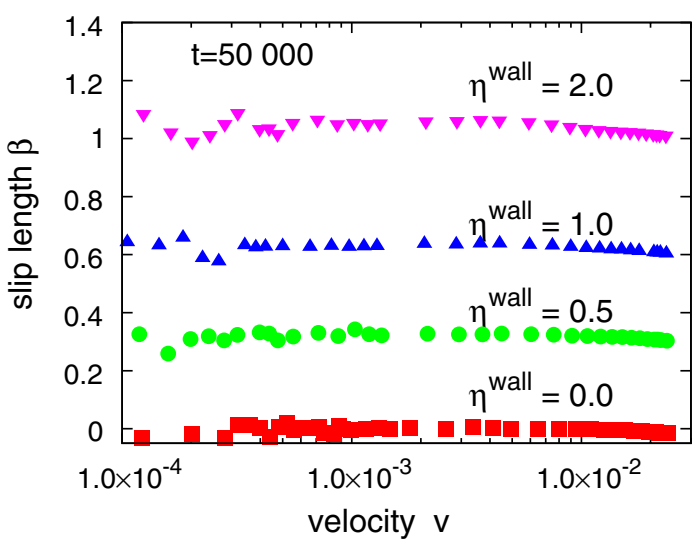

Fig. 1 Slip length $\beta$ versus bulk velocity $v$ for different fluid-wall interactions $\eta^{\text {wall }}$. $\beta$ is independent of $v$ and only depends on $\eta^{\text {wall }}$ (Harting et al. 2006). All units are expressed in lattice units throughout this article, if not stated otherwise

shows a typical example of a simulation setup: Poiseuille flow between two rough surfaces. The surface is generated using a random number generator to randomly choose the height of the obstacles at every discrete surface position. As can be observed in the figure, the stream lines of the flow are getting disturbed or trapped between the obstacles at the surfaces. In this section, we show that an apparent boundary slip can have its origin in the misleading assumption of perfectly smooth boundaries.

The influence of surface variations on the slip length $\beta$ has been investigated by numerous authors. It was demonstrated by Richardson that roughness leads to higher drag forces and thus to no-slip on macroscopic scales. He has shown that if on a rough surface even a full-slip boundary condition is applied, one obtains a flow speed reduction near the boundary resulting in a macroscopic no-slip boundary condition (Richardson 1973). An experimental confirmation was later presented by by McHale and Newton (2004). The MD simulations of Couette flow between sinusoidal walls have been presented by Jabbarzadeh et al. (2000). They found that slip appears for roughness amplitudes smaller than the molecular length scale (Jabbarzadeh et al. 2000). Sbragaglia et al. applied the LB method to simulate fluids in the vicinity of microstructured hydrophobic surfaces (Sbragaglia et al. 2006), Al-Zoubi et al. demonstrated that the LB method is well applicable to reproduce known flow patterns in sinusoidal channels (Al-Zoubi and Brenner 2008), and Varnik et al. (Varnik and Raabe 2006; Varnik et al. 2006) have shown that even in small geometries, rough channel surfaces can cause flow to become turbulent.

Recently, we presented the idea of an effective wall for rough channel surfaces (Kunert and Harting 2007). Here, we investigate the influence of different types of roughness on the position of the effective boundary. Further, we show how the effective boundary depends on the distribution of 


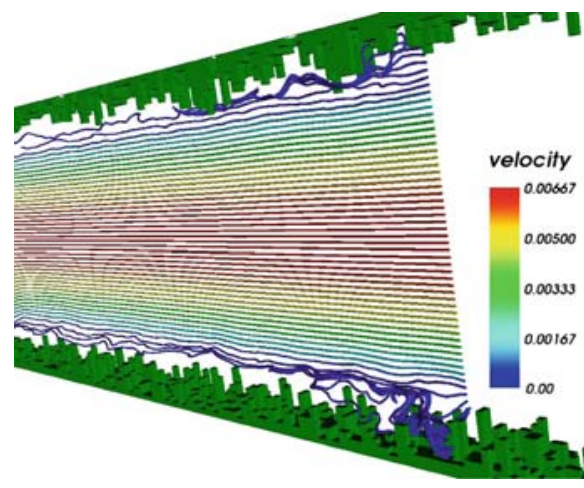

Fig. 2 Left A typical simulated system. Poiseuille flow between two rough surfaces showing random surface variations. Streamlines depict a two dimensional cut and illustrate the parabolic velocity profile. This profile is distorted in the vicinity of the rough surfaces (Kunert and Harting 2008b). Right The effective boundary height $h_{\mathrm{eff}}$ is found

the roughness elements, and how roughness and hydrophobicity interact with each other (Kunert and Harting 2008b). Lecoq et al. (2004) performed experiments with well-defined roughness, and developed a theory to predict the position of the effective boundary. In the experiments, they utilized a laser interferometer to measure the trajectory of a colloidal sphere, and, thereby, determined the lubrication force and an effective boundary position. The used geometry consists of grooves with a triangular profile. For a theoretical description, the boundary is expressed in a Fourier series that gives the boundary condition for the Laplace equation. As a result, an effective boundary can be derived by a fast converting series.

In this article, we revise our previous achievements and compare them with the theoretical and experimental results of Lecoq et al. (2004).

Again, Poiseuille flow measurements are utilized to investigate the effect of interest. The rough surfaces are characterized by the highest point of one plane $\left(h_{\max }\right)$, the position of the deepest valley $\left(h_{\min }\right)$, and the arithmetic average of all surface heights giving the average roughness $R_{a}$. In the case of symmetrical distributions, we get $R_{a}=h_{\max } / 2$.

The position of the effective boundary $h_{\text {eff }}$ can be found by fitting the parabolic flow profile via the distance $d_{\text {eff }}$. With $\beta$ set to 0 , we obtain the no-slip case. In order to obtain an average value for the effective distance between the planes $d_{\text {eff }}$, a sufficient number of individual profiles at different positions $z$ are taken into account. The $d_{\text {eff }}$ so found gives the position of the effective boundary, and the effective height $h_{\text {eff }}$ of the rough surface is then defined by $d_{\text {max }}-d_{\text {eff }}$ (see Fig. 2, left).

We show that the position of the effective boundary height is depending on the shape of the roughness elements, i.e., for strong surface distortions, it is between 1.69 and 1.90 times the average height of the roughness $R_{a}=h_{\max } / 2$

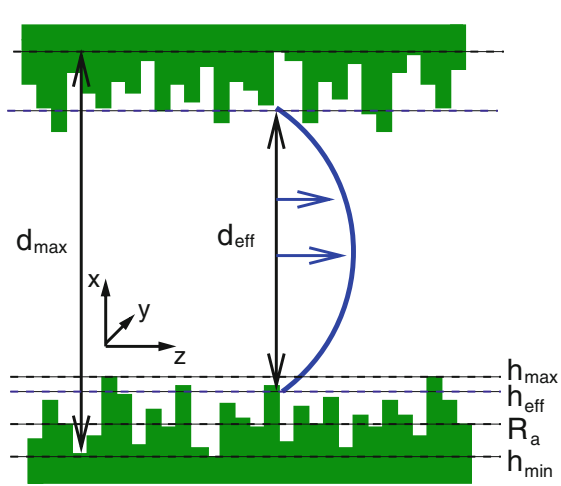

between the deepest valley at $h_{\min }$ and the highest peak at $h_{\max }$. It corresponds to an effective channel width $d_{\text {eff. }} R_{a}$ denotes the average roughness, and the maximum distance between the plates $d_{\max }$ is kept constant (Kunert and Harting 2008b)

(Kunert and Harting 2007). In Fig. 3, we plot the effective boundary positions of different geometries, i.e., randomly distributed grooves with a square profile and grooves with a triangular profile. The results for the triangular ones match with the theoretical value of Lecoq et al. (2004) for a similar geometry.

By adding an additional distance between roughness elements, $h_{\text {eff }}$ decreases slowly, so that the maximum height is still the leading parameter. We are also able to simulate flow over surfaces generated from AFM data of gold-coated glass used in microflow experiments by Vinogradova and Yakubov (2006). We find that the height distribution of such a surface is Gaussian and that a randomly arranged surface with a similar distribution gives the same result for the position of the effective boundary although in this case the heights are not correlated (Fig. 4).

We can tune the width of the distribution $\sigma$ and the average height $R_{a}$. By scaling $\sigma$ with $R_{a}$, we obtain geometrically similar geometries. This similarity is important because the effective height, $h_{\text {eff }}$, scales with the average

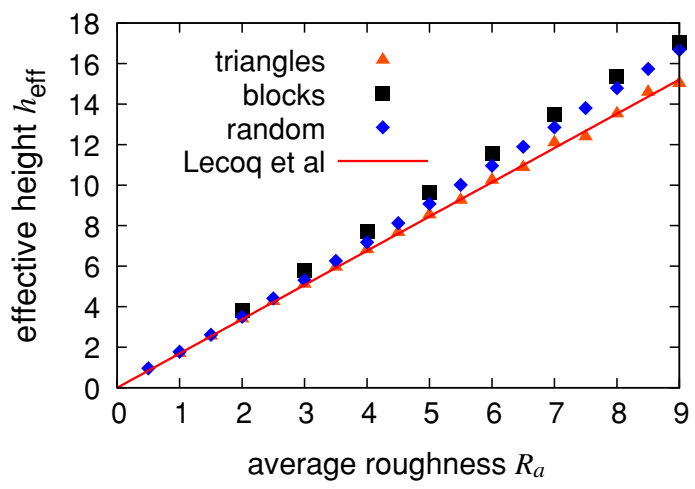

Fig. 3 Simulated effective height $h_{\mathrm{eff}}$ versus $R_{a}$ for different surface geometries. The triangular shape matches the theoretical results of Lecoq et al. (2004) for a similar geometry 
roughness in the case of geometrical similarity (Kunert and Harting 2007). We investigate Gaussian-distributed heights with different widths $\sigma$ and find that the height of the effective wall depends linearly on $\sigma$ in the observed range (Kunert and Harting 2008b). Further, we find that the slip diverges as the amplitude of the roughness increases and the flow field gets more restricted, which highlights the importance of a proper treatment of surface variations in very confined geometries (Kunert and Harting 2007).

\section{Structured surfaces with entrapped microbubbles}

A natural continuation of our previous studies on roughness-induced apparent boundary slip and the collaboration mentioned above is the analysis of flow along superhydrophobic surfaces (Hyväluoma and Harting 2008). While in typical experiments, slip lengths of a few tens of nanometers can be observed, it would be preferable for technical applications to increase the throughput of fluid in a microchannel, i.e., to obtain substantially larger slip. Superhydrophobic surfaces are promising in this context, since it has been recently predicted (Cottin-Bizonne et al. 2003) and experimentally reported (Perot and Rothstein 2004) that the so-called Fakir effect or Cassie state considerably amplifies boundary slippage. Using highly rough hydrophobic surfaces, such a situation can be achieved. Instead of entering the area between the rough surface elements, the liquid remains at the top of the roughness and traps air in the interstices. Thus, a very small liquid-solid contact area is generated.

Steinberger et al. utilized surfaces patterned with a square array of cylindrical holes to demonstrate that gas bubbles present in the holes may cause a reduced slip (Steinberger et al. 2007). Numerically, they found even

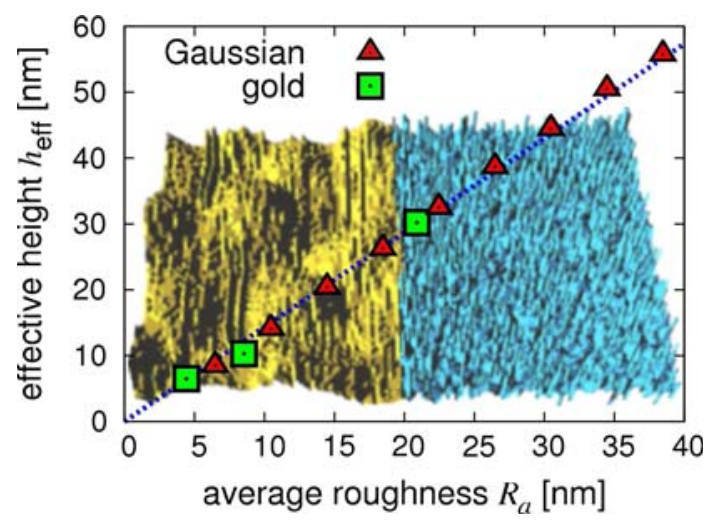

Fig. 4 Simulated effective height $h_{\text {eff }}$ versus $R_{a}$ for gold-coated glass surfaces and a randomly generated surface with Gaussian distributed heights. The background image shows the gold coated glass surface on the left and the artificially generated structure on the right (Kunert and Harting 2007) negative slip lengths for flow over such bubble mattresses, i.e., the effective no-slip plane is inside the channel, and the bubbles increase the flow resistance. In this section, we consider negative slip lengths on bubble surfaces and also discuss the question of shear-rate dependent slip. In particular, we show that microbubbles can generate a shearrate dependence.

Our simulations utilize the single component multiphase LB model by Shan and Chen (1994), which enables simulations of liquid-vapor systems with surface tension. We are not aware of further lattice Boltzmann simulations to study the flow over a bubble mattress. However, a number of authors have applied various LB multiphase and multicomponent models to study the properties of droplets on chemically patterned and superhydrophobic surfaces (Kusumaatmaja et al. 2006; Kusumaatmaja and Yeomans 2007; Pirat et al. 2008; Hyväluoma et al. 2007). The flow in our system is confined between two parallel walls. One of the walls is patterned with holes and vapor bubbles are trapped to these holes. The other wall is smooth and moved with velocity $u_{0}$. Steinberger et al. (2007) presented finiteelement simulations of flow over rigid "bubbles" by applying slip boundaries at static bubble surfaces. The LB method allows the bubbles to deform if the viscous forces are high enough compared to the surface tension. We are also interested in how surface patterning affects the slip properties of these surfaces, and how bubbles could be utilized to develop surfaces with special properties for microfluidic applications (Hyväluoma and Harting 2008).

The distance between walls is $d=1 \mu \mathrm{m}$ (40 lattice nodes) in all the simulations, and the area fraction of holes is 0.43 . A unit cell of the regular array is included in a simulation, and periodic boundary conditions are applied at domain boundaries. The bubbles are trapped into holes by using different wettabilities for boundaries in contact with the main channel and with the hole. The protrusion angle $\varphi$ (see Fig. 5 for definition) is varied by changing the liquid's bulk pressure. The effective slip length is $\beta=\mu u_{0} / \sigma-d$, where $\sigma=\mu \mathrm{d} v / \mathrm{d} z$ is the shear stress acting on the upper wall and $\mu$ the dynamic viscosity of the liquid.

We investigate the effect of a modified protrusion angle and different surface patterns by using square, rectangular, and rhombic bubble arrays. The cylindrical holes have a radius $a=500 \mathrm{~nm}$, and the area fraction of the holes is equal in all the cases. The shear rate is such that the Capillary number $C a=\mu a G_{s} / \gamma=0.16$. Here, $G_{s}$ and $\gamma$ are the shear rate and surface tension, respectively. A snapshot of a simulation is shown in the left part of Fig. 5, and the slip lengths obtained are shown in the right part. The observed behavior is similar to that reported in Steinberger et al. (2007), where a square array of holes was studied. In particular, we observe that when $\varphi$ is large enough, $\beta$ becomes negative. Moreover, when the protrusion angle 

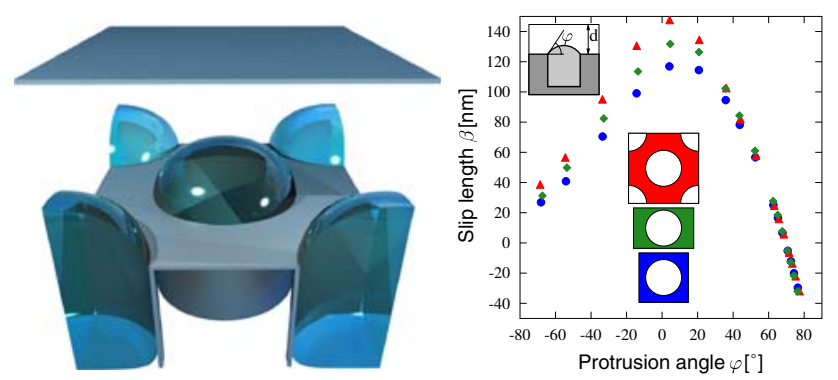

Fig. 5 A visualization of the simulation setup (left) the lower surface is patterned with holes, while the upper surface is moved with velocity $u_{0}$. Right the slip length $\beta$ as a function of protrusion angle $\varphi$. A unit cell of each array is shown in insets, and corresponding results are given by triangles (rhombic array), diamonds (rectangular array), and circles (square array). The inset in the top-left corner shows the definition of $\varphi$ (Hyväluoma and Harting 2008)

equals zero, the slip length is maximized, and the highest possible throughput in a microchannel is obtained. The behavior of the slip length can be explained by thinking of an increased surface roughness if the protrusion angle is greater or less than zero. Since the area fraction of the bubbles is the same in all the three cases, our results clearly indicate that slip properties of the surface can be tailored not only by changing the protrusion angle but also by the array geometry. In this study, the highest slip lengths are obtained for the rhombic unit cell, and it is an ongoing study to investigate the influence of the array geometry in more detail. Recently, our findings have been confirmed theoretically by Davis and Lauga (2009).

Next, the shear-rate dependence of the slip length is investigated. As the shear rate and, thus, the viscous stresses grow, the bubbles are deformed (see Fig. 6, left) and the flow field is modified. In the central part of Fig. 6, we show the simulated slip length as a function of the Capillary number for three different protrusion angles. The Capillary numbers chosen are in higher end of the experimentally available range. Our results show shear-rate dependent slip, but the behavior is opposite to that found in some experiments: in fact, the slip lengths measured by us decrease with increasing shear due to a deformation of the bubbles. In the experiments, surface force apparatuses are used (see, e.g., Zhu and Granick 2001), where a strong increase in the slip is observed after some critical shear rate. This shear-rate dependence has been explained, e.g., with formation and growth of bubbles (Gennes 2002; Lauga and Brenner 2004). In our simulations, there is no formation or growth of the bubbles as we only simulate a steady case for given bubbles. The experiments on the contrary are dynamic. However, our results indicate that the changes in the flow field which occur due to the deformation of the bubbles cannot be an explanation for the shear-rate dependence observed in some experiments. Our results are consistent with Kunert and Harting (2007) and the previous section, where it is shown that smaller roughness leads to smaller values of a detected slip. In this studied case, the shear reduces the average height of the bubbles, and thus the average scale of the roughness decreases as well.

Finally, we consider a surface patterned with grooves. Cylindrical bubbles protrude to the flow channel from these holes with protrusion angle $\varphi=72^{\circ}$, and the area fraction of slots is 0.53 . We apply shear both parallel and perpendicular to the slots. The slip length is strongly dependent on the flow direction (Hyväluoma and Harting 2008). For parallel flow, the slip length is positive, but for the perpendicular case, it becomes negative. Flow direction affects also greatly on the shear-rate dependence (cf. Fig. 6, right). When flow is parallel to the grooves, no shear-rate dependence is observed, but for the perpendicular case, this dependence is similar to that seen on hole arrays. These results can be understood on the basis of deforming bubbles. For perpendicular flow, the bubbles are able to deform, but for the parallel case, the bubbles retain their shape regardless of the shear rate.

\section{Conclusion}

In this article, we review applications of the lattice Boltzmann method to microfluidic problems. The main focus of this article is on our own research related to the validation of the no-slip boundary condition. By introducing a model for hydrophobic fluid-surface interactions and studying pressure-driven flow in microchannels, we show that an experimentally detected slip can have its origin in hydrophobic interactions, but is constant with varied shear rates and decreases with increasing pressure. Another effect that was not fully understood so far is the influence of surfaces roughness. We are able to apply our simulations to surface data obtained from AFM measurements of experimental samples. We show that ignoring roughness can lead to large errors in a detected slip. In fact, we propose that roughness alone could often be the reason for apparent boundary slip.

Microscale bubbles at surfaces allow to tailor the slip properties of a surface. Such a surface with bubbles may yield negative slip, i.e., increased resistance to flow, if bubbles are strongly protruding to the channel. The lattice Boltzmann simulations capture the deformability of bubbles and thus allow to study the influence of the shear rate on the deformation of the interface and its effect on the measured slip. We find that the slip decreases with increasing shear rate demonstrating that shear-induced bubble deformation cannot explain recent experimental findings where slip increases with increasing shear rate.

In this article, we also demonstrate the suitability of the lattice Boltzmann method for modeling microfluidic applications: in contrast to molecular dynamics, it is able to 

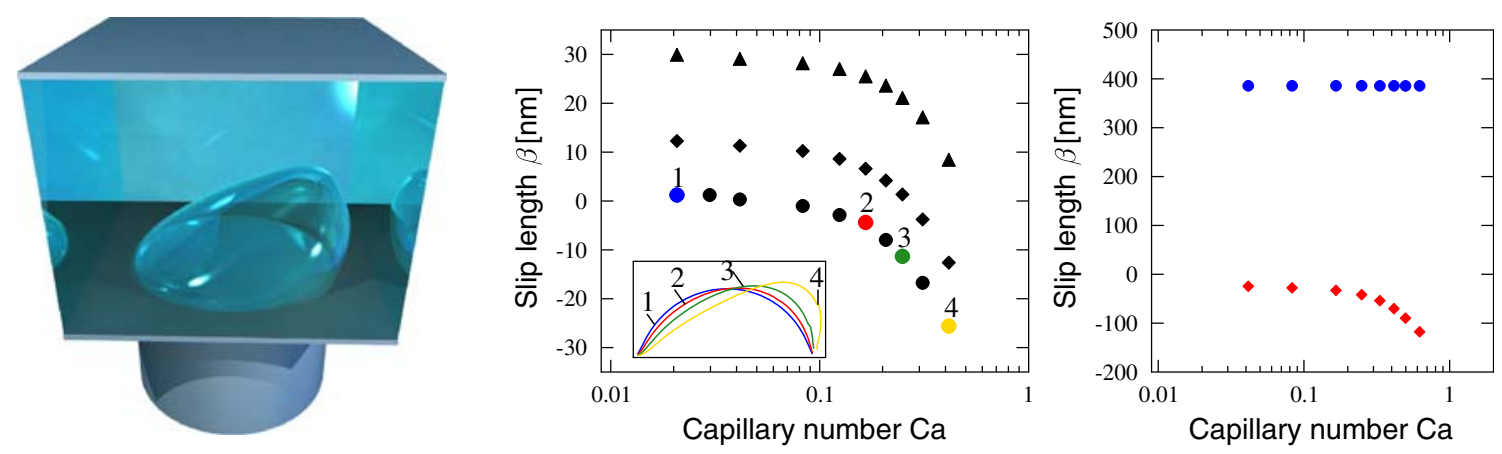

Fig. 6 The left figure shows a snapshot of a bubble deformed by shear flow. In the center, the slip length as a function of the capillary number for a square array of bubbles with three different protrusion angles, $\varphi=63^{\circ}, 68^{\circ}$, and $71^{\circ}$ (from uppermost to lowermost) is shown. The inset shows cross sections of liquid-gas interfaces for four capillary

reach experimentally available time and length scales. This allows one to compare simulation results to experimental data directly as demonstrated in the case of simulations of flow along surface data obtained from AFM measurements of "real" samples.

Acknowledgments This study was financed within the DFG priority program "nano- and microfluidics," the collaborative research center 716 , the German academic exchange service (DAAD), and by the "Landesstiftung Baden-Württemberg." We thank the Neumann Institute for Computing, Jülich and the Scientific Supercomputing Center, Karlsruhe for providing the computing time and technical support for this study. Jyrki Hokkanen (CSC-Scientific Computing Ltd., Espoo, Finland) is gratefully acknowledged for the bubble visualizations. Christian Kunert acknowledges the fruitful discussions with P. Szymczak.

Open Access This article is distributed under the terms of the Creative Commons Attribution Noncommercial License which permits any noncommercial use, distribution, and reproduction in any medium, provided the original author(s) and source are credited.

\section{References}

Al-Zoubi A, Brenner G (2008) Simulating fluid flow over sinusoidal surfaces using the lattice Boltzmann method. Comput Math Appl 55:1365

Ansumali S, Karlin IV (2002) Kinetic boundary conditions in the lattice Boltzmann method. Phys Rev E 66:026311

Barrat JL, Bocquet L (1999) Large slip effect at a nonwetting fluid interface. Phys Rev Lett 82(23):4671

Baudry J, Charlaix E (2001) Experimental evidance for a large slip effect at a nonwetting fluid-solid interface. Langmuir 17:5232

Benzi R, Biferale L, Sbragaglia M, Succi S, Toschi F (2006a) Mesoscopic two-phase model for describing apparent slip in micro-channel flows. Europhys Lett 74:651

Benzi R, Biferale L, Sbragaglia M, Succi S, Toschi F (2006b) Mesoscopic modeling of a two-phase flow in the presence of boundaries: the contact angle. Phys Rev E 74:021509

Bhatnagar PL, Gross EP, Krook M (1954) Model for collision processes in gases. I. Small amplitude processes in charged and neutral one-component systems. Phys Rev 94(3):511 numbers (Hyväluoma and Harting 2008). The right figure shows the slip length as a function of capillary number for a surface with cylindrical bubbles. Circles denote the values for flow parallel to the bubbles, and diamonds for the perpendicular direction

Bocquet L, Barrat JL (2007) Flow boundary conditions from nano- to micro-scales. Soft Matter 3:685

Cheng JT, Giordano N (2002) Fluid flow throug nanometer scale channels. Phys Rev E 65:031206

Chibbaro S, Biferale L, Diotallevi F, Succi S, Binder K, Dimitrov D, Milchev A, Girardo S, Pisignano D (2008) Evidence of thin-film precursors formation in hydrokinetic and atomistic simulations of nano-channel capillary filling. Europhys Lett 84:44003

Choi CH, Westin KJ, Breuer KS (2003) Apparent slip in hydrophilic and hydrophobic microchannels. Phys Fluids 15(10):2897

Churaev NV, Sobolev VD, Somov AN (1984) Slippage of liquids over lyophobic solid surfaces. J Colloid Int Sci 97:574

Cieplak M, Koplik J, Banavar JR (2001) Boundary conditions at a fluid-solid interface. Phys Rev Lett 86:803

Cottin-Bizonne C, Jurine S, Baudry J, Crassous J, Restagno F, Charlaix E (2002) Nanorheology: an investigation of the boundary condition at hydrophobic and hydrophilic interfaces. Eur Phys J E 9:47

Cottin-Bizonne C, Barrat JL, Bocquet L, Charlaix E (2003) Lowfriction flows of liquid at nanopatterned interfaces. Nat Mater 2:237

Cottin-Bizonne C, Barentin C, Charlaix E, Bocquet L, Barrat JL (2004) Dynamics of simple liquids at heterogeneous surfaces: molecular dynamics simulations and hydrodynamic description. Eur Phys J E 15:427

Craig VSJ, Neto C, Williams DRM (2001) Shear dependent boundary slip in an aqueous Newtonian liquid. Phys Rev Lett 87(5): 054504

Davis AMJ, Lauga E (2009) Geometric transition in friction for flow over a bubble mattress. Phys Fluids 21:011701

Gennes P (2002) On fluid/wall slippage. Langmuir 18:3413

Guo Z, Shi B, Zhao TS, Zheng C (2007) Discrete effects on boundary conditions for the lattice Boltzmann equation in simulating microscale gas flows. Phys Rev E 76:056704

Harting J, Kunert C, Herrmann H (2006) Lattice Boltzmann simulations of apparent slip in hydrophobic microchannels. Europhys Lett 75:328-334

Horbach J, Succi S (2006) Lattice Boltzmann versus molecular dynamics simulation of nanoscale hydrodynamic flows. Phys Rev Lett 96:224503

Huang H, Thorne DT, Schaap MG, Sukop MC (2007) Proposed approximation for contact angles in shan-and-chen-type multicomponent multiphase lattice Boltzmann models. Phys Rev E 76:066701

Hyväluoma J, Harting J (2008) Slip flow over structured surfaces with entrapped microbubbles. Phys Rev Lett 100:246001 
Hyväluoma J, Koponen A, Raiskinmäki P, Timonen J (2007) Droplets on inclined rough surfaces. Eur Phys J E 23:289

Jabbarzadeh A, Atkinson JD, Tanner RI (2000) Effect of the wall roughness on slip and rheological properties of hexadecane in molecular dynamics simulation of couette shear flow between two sinusoidal walls. Phys Rev E 61:690

Knudsen M (1909) Experimentelle Bestimmung des Druckes gesättigter Quecksilberdämpfe bei $0^{\circ}$ und höheren Temperaturen. Ann d Phys 29:179

Koplik J, Banavar JR, Willemsen JF (1989) Molecular dynamics of fluid flow at solid-surfaces. Phys Fluids 1:781

Kunert C, Harting J (2007) Roughness induced apparent boundary slip in microchannel flows. Phys Rev Lett 99:176001

Kunert C, Harting J (2008a) On the effect of surfactant adsorption and viscosity change on apparent slip in hydrophobic microchannels. Prog CFD 8:197

Kunert C, Harting J (2008b) Simulation of fluid flow in hydrophobic rough micro channels. Int J Comput Fluid Dyn 22:475

Kusumaatmaja H, Yeomans JM (2007) Modeling contact angle hysteresis on chemically patterned and superhydrophobic surfaces. Langmuir 23:6019

Kusumaatmaja H, Leopoldes J, Dupuis A, Yeomans JM (2006) Drop dynamics on chemicaly patterned surfaces. Europhys Lett 73:740

Lauga E, Brenner MP (2004) Dynamic mechanism for aparrent slip on hydrophobic surfaces. Phys Rev E 70:026311

Lauga E, Brenner MP, Stone HA (2005) Microfluidics: the no-slip boundary condition, in handbook of experimental fluid dynamics, chap 15. Springer, Berlin

Lecoq N, Anthore R, Cickhocki B, Szymczak P, Feuillebois F (2004) Drag force on a sphere moving towards a corrugated wall. J Fluid Mech 513:247

McHale G, Newton MI (2004) Surface roughness and interfacial slip boundary condition for quarzcrystal microbalances. J Appl Phys 95:373

Nagayama G, Cheng P (2004) Effects of interface wettability on microscale flow by molecular dynamics simulation. Int $\mathrm{J}$ Heat Mass Transf 47:501

Navier CLMH (1823) Mémoire sur les lois du mouvement de fluids. Mem Acad Sci Inst Fr 6:389

Neto C, Craig VSJ, Williams DRM (2003) Evidence of sheardependent boundary slip in Newtonian liquids. Eur Phys J E 12:71

Neto C, Evans DR, Bonaccurso E, Butt HJ, Craig VSJ (2005) Boundary slip in Newtonian liquids: a review of experimental studies. Rep Prog Phys 68:2859

Nie X, Doolen GD, Chen S (2002) Lattice-Boltzmann simulations of fluid flows in MEMS. J Stat Phys 107(112):279

Niu XD, Shu C, Chew YT (2004) A lattice Boltzmann BGK model for simulation of micro flows. Europhys Lett 67:600

Perot OJ, Rothstein JP (2004) Laminar drag reduction in microchannels using ultrahydrophobic surfaces. Phys Fluids 16:4635

Pirat C, Sbragaglia M, Peters AM, Borkent BM, Lammertink RGH, Wesseling M, Lohse D (2008) Multiple time scale dynamics in the breakdown of superhydrophobicity. Europhys Lett 81:66002

Priezjev NV, Darhuber A, Troian S (2005) Slip behavior in liquid films on surfaces of patterned wettability: comparison between continuum and molecular dynamics simulations. Phys Rev E 71:041608

Richardson S (1973) On the no-slip boundary condition. J Fluid Mech 59:707

Sbragaglia M, Succi S (2005) Analytical calculation of slip flow in lattice Boltzmann models with kinetic boundary conditions. Phys Fluids 17:093602
Sbragaglia M, Benzi R, Biferale L, Succi S, Toschi F (2006) Surface roughness-hydrophobicity coupling in microchannel and nanochannel flows. Phys Rev Lett 97:204503

Shan X, Chen H (1993) Lattice Boltzmann model for simulating flows with multiple phases and components. Phys Rev E 47(3):1815

Shan X, Chen H (1994) Simulation of nonideal gases and liquid-gas phase transitions by the lattice Boltzmann equation. Phys Rev E 49(4):2941

Sofonea V, Sekerka RF (2005) Diffuse-reflection boundary conditions for a thermal lattice Boltzmann model in two dimensions: evidence of temperature jump and slip velocity in microchannels. Phys Rev E 71:066709

Steinberger A, Cottin-Bizonne C, Kleimann P, Charlaix E (2007) High friction on a bubble mattress. Nat Mater 6:665

Succi S (2001) The lattice Boltzmann equation for fluid dynamics and beyond. Oxford University Press, Oxford

Succi S (2002) Mesoscopic modeling of slip motion at fluid-solid interfaces with heterogeneous catalysis. Phys Rev Lett 89(6): 064502

Tabeling P (2005) Introduction to microfluidics. Oxford University Press, Oxford

Tang GH, Tao WQ, He YL (2005) Lattice Boltzmann method for gaseous microflows using kinetic theory boundary conditions. Phys Fluids 17:058101

Thompson PA, Robbins MO (1990) Shear flow near solids: epitaxial order and flow boundary conditions. Phys Rev A 41:6830

Thompson PA, Troian S (1997) A general boundary condition for liquid flow at solid surfaces. Nature 389:360

Tretheway DC, Meinhart CD (2002) Apparent fluid slip at hydrophobic microchannel walls. Phys Fluids 14:L9

Tretheway DC, Meinhart CD (2004) A generating mechanism for apparent fluid slip in hydrophobic microchannels. Phys Fluids 16(5): 1509

Tretheway D, Zhu L, Petzold L, Meinhart C (2002) Examination of the slip boundary condition by $\mu$-PIV and lattice Boltzmann simulation. In 2002 ASME international mechanical engineering congress \& exposition, New Orleans, Louisiana

Varnik F, Raabe D (2006) Scaling effects in microscale fliuid flows at rough solid surfaces. Model Simul Mater Sci Eng 14:857

Varnik F, Dorner D, Raabe D (2006) Roughness-induced flow instability: a lattice Boltzmann study. J Fluid Mech 573:191

Vinogradova OI (1995) Drainage of a thin film confined between hydrophobic surfaces. Langmuir 11:2213

Vinogradova OI (1996) Possible implications of hydrophopic slippage on the dynamic measurements of hydrophobic forces. J Phys Condens Matter 8:9491

Vinogradova OI, Yakubov GE (2003) Dynamic effects on force mesurements. 2. Lubrication and the atomic force microscope. Langmuir 19:1227

Vinogradova OI, Yakubov GE (2006) Surface roughness and hydrodynamic boundary conditions. Phys Rev E 73:045302(R)

Zhang J, Kwok DY (2004) Apparent slip over a solid-liquid interface with a no-slip boundary condition. Phys Rev E 70:056701

Zhu Y, Granick S (2001) Rate-dependent slip of Newtonian liquid at smooth surfaces. Phys Rev Lett 87:096105

Zhu L, Tretheway D, Petzold L, Meinhart C (2005) Simulation of fluid slip at $3 \mathrm{~d}$ hydrophobic microchannel walls by the lattice Boltzmann method. J Comput Phys 202:181 